GANIT J. Bangladesh Math. Soc. (ISSN 1606-3694) 32 (2012) 43-54

\title{
A NUMERICAL SIMULATION OF SEVERE CYCLONE SIDR-2007
}

\author{
Nikhil Chandra Sarkar ${ }^{1}$, Anowarul Islam ${ }^{2}$ and Sujit Kumar Debsarma ${ }^{3}$ \\ ${ }^{1}$ Department of Mathematics, International University of Business \\ Agriculture and Technology, Dhaka-1230, Bangladesh \\ ${ }^{2}$ Department of Mathematics, Shahjalal University of Science and Technology \\ Sylhet-3114, Bangladesh \\ ${ }^{3}$ Theoretical Division, SAARC Meteorological Research Centre Dhaka-1207, Bangladesh \\ E-mail: nikhil_cs48@yahoo.com
}

Received 21-10-2011 Accepted 03-11-2012

\begin{abstract}
The most severe tropical cyclones are formed over the Bay of Bengal because of its complex geometric structure and shallow coastal bathymetry. These natural disasters significantly impact our lives as well as the environment. To quantify the impact of such disasters, we analyzed data pertaining to the severe cyclone Sidr-2007, considering the cyclone's duration and angle of land fall, as well as the bathymetry of the near-coastal zones. We propose a reliable hydrodynamic model to simulate the severity of such cyclones. The model's reliability is verified by comparison of the numerical results with actual storm-surge data. Our model requires meteorological and hydrological inputs, including the time-dependent positions of the storm centre, the maximum wind radius, as well as pressure-drop and bathymetry data. The model results are in good agreement with the reported data.
\end{abstract}

Keywords: Bathymetry, Bay of Bengal, Coastal zone, Cyclones, Simulation, Storm Surges

\section{Introduction}

The Bay of Bengal is frequently affected by storm surges associated with tropical cyclones. Storm surges-i.e., extreme overflows of sea water onto coastal regions by the force of strong winds associated with low-pressure weather systems-are long waves that increase both the sea water level and the tides during cyclones. They may last from a few minutes to several days, depending on the cyclone's size and speed of movement. Statistics show that approximately 5\% of the world's tropical cyclones form over the Bay of Bengal. On average, five to six storms form in this region every year. Of all countries surrounding the Bay of Bengal, Bangladesh suffers most from storm surges caused by tropical cyclones, almost every year. Eighty percent of the global casualties due to storm surges occur in Bangladesh because of the complex coastal geometry of the Bay of Bengal's northern tip. 
Different aspects of the procedure and the developments involved are reported in the papers by Flather (1976) in [11], Heaps et al. (1979) in [14]. Some attempts have been made to develop numerical simulations of storm surges associated with tropical cyclones over the Bay of Bengal and the Arabian Sea. Storm-surge modeling studies of the Bay of Bengal include those of Das (1981) in [5], Das et al. (1983) in [6], Dube et al. (1984) in [10], Gosh et al. (1983) in [12], Johns (1981) in [19], and Johns et al. (1983) in [20]; for comprehensive reviews of previous studies, see also Das (1980) in [4], Dube and Singh (1982) in [9], Ali (1996) in [1], Ali et al. (1997) in [2], Debsarma $(1997,2009)$ in [7] and [8], Sarkar (2011) in [17], Chittibabu (1999) in [3], Roy (1999) in [16], and Hoque (2008) in [15]. Very limited simulation studies have been performed for storm surges in the Arabian Sea. To our knowledge, probably the only simulations of storm surges along the western coast of India are those of Dube et al. (1982) in [9], Ghosh and Chandar (1983) in [13], and Sinha et al. (1984) in [18].

\section{Hydrodynamic storm-surge Model}

Numerical computation has become a powerful and popular tool to study oceanographic natural disasters and coastal processes, among others. The Indian Institutes of Technology (IIT) Storm Surge Model is a state-of-the-art numerical hydrodynamic storm-surge model suite. It uses the custom-developed GMT graphics software to visualize the results. A dynamical storm model is adopted for computation of surface winds and ocean currents associated with cyclonic storms. The required meteorological and hydrological inputs are the position of the storm center as a function of time and the radius of maximum wind, as well as pressure-drop and bathymetry data. The number of iterations (which depends on the time resolution required), the latitude of the open sea, the East-West and North-South extents of the area(s) of interest, and the number of storm positions are also important inputs.

The parameters adopted for the simulations performed in this paper are based on actual data of the severe cyclone Sidr-2007. They are summarized below.

- $\quad$ Number of East-West grid points

- Number of North-South grid points

- Storm track's starting point

- Storm track's end point

- Duration of model run

- $\quad$ Time step (DT)

- $\quad$ Number of iterations $(K S)$

- $\quad$ Radius of maximum wind $\left(R_{\max }\right)$

- $\quad$ Pressure drop
331

154

$18^{\circ} \mathrm{N}, \quad 89.20^{\circ} \mathrm{E}$

$22^{\circ} \mathrm{N}, 89.80^{\circ} \mathrm{E}$

$18 \mathrm{hr}$

$60 \mathrm{~s}$

$1080(K S=D T \times$ duration in $\mathrm{hr})$

$40 \mathrm{~km}$

$64 \mathrm{hP}$

\section{Governing equations}

The basic hydrodynamic equation of continuity for dynamical processes at sea is given by 


$$
\frac{\partial u}{\partial x}+\frac{\partial v}{\partial y}+\frac{\partial w}{\partial z}=0
$$

The momentum equations can be written in terms of the $u, v$, and $w$ components as

$$
\begin{aligned}
& \frac{\partial u}{\partial t}+u \frac{\partial u}{\partial x}+v \frac{\partial u}{\partial y}+w \frac{\partial u}{\partial z}=-\frac{1}{\rho} \frac{\partial p}{\partial x}+2 \omega v \sin \varphi-2 \omega w \cos \varphi+F_{x} \\
& \frac{\partial v}{\partial t}+u \frac{\partial v}{\partial x}+v \frac{\partial v}{\partial y}+w \frac{\partial v}{\partial z}=-\frac{1}{\rho} \frac{\partial p}{\partial y}-2 \omega u \sin \varphi+F_{y} \text { and } \\
& \frac{\partial w}{\partial t}+u \frac{\partial w}{\partial x}+v \frac{\partial w}{\partial y}+w \frac{\partial w}{\partial z}=-\frac{1}{\rho} \frac{\partial p}{\partial z}+2 \omega u \cos \varphi-g
\end{aligned}
$$

For large-scale meteorology, $\omega \ll u, v$, so neglecting the $\omega$-related terms is justified, i.e.,

$\frac{\partial u}{\partial x}+\frac{\partial v}{\partial y}+\frac{\partial w}{\partial z}=0$

$\frac{\partial u}{\partial t}+u \frac{\partial u}{\partial x}+v \frac{\partial u}{\partial y}+w \frac{\partial u}{\partial z}-f v=-\frac{1}{\rho} \frac{\partial p}{\partial x}+\frac{1}{\rho} \frac{\partial \tau_{x}}{\partial z}$

$\frac{\partial v}{\partial t}+u \frac{\partial v}{\partial x}+v \frac{\partial v}{\partial y}+w \frac{\partial v}{\partial z}+f u=-\frac{1}{\rho} \frac{\partial p}{\partial y}+\frac{1}{\rho} \frac{\partial \tau_{y}}{\partial z}$ and

$\frac{\partial w}{\partial t}+u \frac{\partial w}{\partial x}+v \frac{\partial w}{\partial y}+w \frac{\partial w}{\partial z}=-\frac{1}{\rho} \frac{\partial p}{\partial z}-g=0$

where $\omega$ is the angular speed of rotation of the earth, and $f=2 \omega \sin \varphi$ is the Coriolis parameter, and $u, v$, and $w$ are the average Reynolds velocity components in the $x, y$, and $z$ directions, respectively, defined as $u=\dot{u}+\bar{u}, v=v^{\prime}+\bar{v}$, and $w=w^{\prime}+\bar{w}$, where $u^{\prime}, v^{\prime}$, and $w^{\prime}$ are the fluctuating (turbulence) velocity components, and $\bar{u}, \bar{v}$, and $\bar{w}$ are the average velocity components in the $x, y$, and $z$ directions, respectively. Here $p$ denotes pressure, $t$ time, $\rho$ the density of sea water (which is supposed to be incompressible and homogeneous), $g$ is the acceleration due to gravity, and $\tau_{x}$ and $\tau_{y}$ are the $x$ and $y$ components of the frictional (Reynolds) stress, respectively. We neglect the molecular viscosity. Using scale analysis, the vertical momentum equation (8) reduces to the hydrostatic pressure approximation,

$$
\frac{\partial p}{\partial z}=-\rho g
$$

We represent the displaced position of the free surface and the position of the sea floor by $z=\zeta(x, y, t)$ and $z=-h(x, y)$, respectively, so that the total depth of the water level is $\zeta+h$. During a storm period, the upper surface stress is generated by the circulatory wind of the storm system, while the bottom stress acts as dissipation term, which is known as bottom friction. The terms $\tau_{x}$ and $\tau_{y}$ are included to model the vertical turbulent diffusion. We denote the $x$ and $y$ components of the surface stress as $T_{x}$ and $T_{y}$, respectively, and adopt the same convention for the bottom friction, $F_{x}$ and $F_{y}$. Atmospheric pressure is denoted as $P_{\mathrm{a}}$. The bottom and surface conditions are then given by (at the bottom)

$u=v=w=0$ and $\quad\left(\tau_{x}, \tau_{y}\right)=\left(F_{x}, F_{y}\right) \quad$ at $z=-h(x, y)$

(at the free surface)

$\left(\tau_{x}, \tau_{y}\right)=\left(T_{x}, T_{y}\right), P=P_{\mathrm{a}}$ and $w=\frac{\partial \zeta}{\partial t}+u \frac{\partial \zeta}{\partial x}+v \frac{\partial \zeta}{\partial y} \quad$ at $z=\zeta(x, y, t)$ 
Condition (11) is known as the kinematic surface condition. It expresses the fact that the free surface materially follows the fluid. Equations (5), (6), (7), and (9) can be solved in principle, but the procedure would be laborious because of the presence in the equations of the vertical coordinate. Unlike for atmospheric problems, a boundary layer would need to be designed both at the top and the bottom of the integration domain. There is currently insufficient knowledge about the flow characteristics in such boundary layers. To overcome this difficulty, a simplification is generally introduced by integrating the governing equations vertically. The unknown dependent variables are then the water transport (or mean) current and the surface height. This procedure is commonly adopted for storm-surge computations, because the water level is of primary importance.

Upon vertically integrating Equations (5) to (7) from $z=-h$ to $z=\zeta$ and using boundary conditions (9) to (11), in addition to adopting the simplification discussed above, one obtains

$$
\begin{aligned}
& \frac{\partial \zeta}{\partial t}+\frac{\partial}{\partial x}[(\zeta+h) \bar{u}]+\frac{\partial}{\partial y}[(\zeta+h) \bar{v}]=0 \\
& \frac{\partial \bar{u}}{\partial t}+\bar{u} \frac{\partial \bar{u}}{\partial x}+\bar{v} \frac{\partial \bar{u}}{\partial y}-f \bar{v}+\frac{1}{(\zeta+h)} {\left[\frac{\partial}{\partial x}(\zeta+h)\left(\overline{u^{2}}-\bar{u}^{2}\right)+\frac{\partial}{\partial y}(\zeta+h)(\overline{u v}-\bar{u} \bar{v})\right] } \\
&=-g \frac{\partial \zeta}{\partial x}-\frac{1}{\rho} \frac{\partial P_{\mathrm{a}}}{\partial x}+\frac{1}{(\zeta+h) \rho}\left[T_{x}-F_{x}\right] \text { and } \\
& \frac{\partial \bar{v}}{\partial t}+\bar{u} \frac{\partial \bar{v}}{\partial x}+\bar{v} \frac{\partial \bar{v}}{\partial y}+f \bar{u}+\frac{1}{(\zeta+h)} {\left[\frac{\partial}{\partial x}(\zeta+h)(\overline{u v}-\bar{u} \bar{v})+\frac{\partial}{\partial y}(\zeta+h)\left(\overline{v^{2}}-\bar{v}^{2}\right)\right] } \\
&=-g \frac{\partial \zeta}{\partial y}-\frac{1}{\rho} \frac{\partial P_{\mathrm{a}}}{\partial y}+\frac{1}{(\zeta+h) \rho}\left[T_{y}-F_{y}\right]
\end{aligned}
$$

where over bars denote depth-averaged values. For example,

$$
\begin{aligned}
& (\bar{u}, \bar{v})=\frac{1}{(\zeta+h)} \int_{-h}^{\zeta}(u, v) \mathrm{d} z, \\
& \left(\overline{u^{2}}, \overline{v^{2}}\right)=\frac{1}{(\zeta+h)} \int_{-h}^{\zeta}\left(u^{2}, v^{2}\right) \mathrm{d} z \text { and } \\
& (\overline{u v})=\frac{1}{(\zeta+h)} \int_{-h}^{\zeta} u v \mathrm{~d} z
\end{aligned}
$$

In most storm-surge simulation models, the nonlinear advective terms are neglected mainly on the basis of scale analysis. This is justifiable when the characteristic amplitude of the surge is significantly lower than the characteristic depth of the basin. In shallowwater regions, particularly at the head of the Bay of Bengal, the nonlinear terms are of special importance and must be retained in the formulation. However, retention in Equation (13) of terms such as $\left(\overline{u^{2}}, \bar{u}^{2}\right)$ leads to a fundamental difficulty, since they cannot be evaluated within the framework of a vertically integrated model. In many welldocumented applications of the nonlinear, vertically integrated Equations (12) to (14), assumptions are usually typified by

$$
\begin{aligned}
& \overline{u^{2}}-\bar{u}^{2}=0 \\
& \overline{v^{2}}-\bar{v}^{2}=0 \text { and } \\
& \overline{u v}-\bar{u} \bar{v}=0
\end{aligned}
$$


This is equivalent to saying that the currents $\bar{u}$ and $\bar{v}$ do not vary significantly in the vertical direction and that the flow is dominated by the midstream flow. The validity of Equation (18) has been demonstrated by Johns (1981) in [19]. In the latter article, the author found that,

for all $t$,

$$
0.996<\left(\frac{\bar{u}^{2}}{\overline{u^{2}}}\right)<1.04
$$

In addition, a parameterization of the bottom stress must be adopted in terms of the depth-averaged current. This is frequently done by using the conventional quadratic law,

$$
\begin{aligned}
& F_{x}=\rho c_{\mathrm{f}} \bar{u}\left(\bar{u}^{2}+\bar{v}^{2}\right)^{1 / 2} \\
& F_{y}=\rho c_{\mathrm{f}} \bar{v}\left(\bar{u}^{2}+\bar{v}^{2}\right)^{1 / 2}
\end{aligned}
$$

where $c_{\mathrm{f}}=2.6 \times 10^{-3}$ is an empirical bottom-friction coefficient.

However, substituting the values from Equations (18) and (20) into Equations (13) and (14), we obtain (the overbars have been dropped for convenience)

$$
\begin{aligned}
& \frac{\partial u}{\partial t}+u \frac{\partial u}{\partial x}+v \frac{\partial u}{\partial y}-f v=-g \frac{\partial \zeta}{\partial x}-\frac{1}{\rho} \frac{\partial P_{\mathrm{a}}}{\partial x}+\frac{1}{(\zeta+h)}\left[\frac{T_{x}}{\rho}-c_{\mathrm{f}} u\left(u^{2}+v^{2}\right)^{1 / 2}\right] \\
& \frac{\partial v}{\partial t}+u \frac{\partial v}{\partial x}+v \frac{\partial v}{\partial y}+f u=-g \frac{\partial \zeta}{\partial y}-\frac{1}{\rho} \frac{\partial P_{\mathrm{a}}}{\partial y}+\frac{1}{(\zeta+h)}\left[\frac{T_{y}}{\rho}-c_{\mathrm{f}} u\left(u^{2}+v^{2}\right)^{1 / 2}\right]
\end{aligned}
$$

For numerical treatment, it is convenient to express Equations (21) and (22) in flux form,

$$
\begin{gathered}
\frac{\partial}{\partial t}[(\zeta+h) u]+\frac{\partial}{\partial x}[(\zeta+h) u u]+\frac{\partial}{\partial y}[(\zeta+h) u v]-f(\zeta+h) v \\
=-g(\zeta+h) \frac{\partial \zeta}{\partial x}-\frac{1}{\rho}(\zeta+h) \frac{\partial P_{\mathrm{a}}}{\partial x}+\frac{T_{x}}{\rho}-c_{\mathrm{f}} u\left(u^{2}+v^{2}\right)^{1 / 2} \\
\frac{\partial}{\partial t}[(\zeta+h) v]+\frac{\partial}{\partial x}[(\zeta+h) u v]+\frac{\partial}{\partial y}[(\zeta+h) v v]+f(\zeta+h) u \\
=-g(\zeta+h) \frac{\partial \zeta}{\partial y}-\frac{1}{\rho}(\zeta+h) \frac{\partial P_{a}}{\partial y}+\frac{T_{x}}{\rho}-c_{\mathrm{f}} v\left(u^{2}+v^{2}\right)^{1 / 2}
\end{gathered}
$$

Equations (13), (24), and (25) can be simplified to

$$
\begin{aligned}
& \frac{\partial \zeta}{\partial t}+\frac{\partial \widetilde{u}}{\partial x}+\frac{\partial \tilde{v}}{\partial y}=0 \\
& \frac{\partial \widetilde{u}}{\partial t}+\frac{\partial}{\partial x}(u \tilde{v})+\frac{\partial}{\partial y}(u \tilde{v})-f \tilde{v} \\
& =-g(\zeta+h) \frac{\partial \zeta}{\partial x}-\frac{1}{\rho}(\zeta+h) \frac{\partial P_{\mathrm{a}}}{\partial x}+\frac{T_{x}}{\rho}-\frac{c_{\mathrm{f}} \widetilde{u}}{(\zeta+h)}\left(u^{2}+v^{2}\right)^{\frac{1}{2}} \\
& \frac{\partial \tilde{v}}{\partial t}+\frac{\partial}{\partial x}(u \tilde{v})+\frac{\partial}{\partial y}(u \tilde{v})+f \tilde{u} \\
& =-g(\zeta+h) \frac{\partial \zeta}{\partial y}-\frac{1}{\rho}(\zeta+h) \frac{\partial P_{\mathrm{a}}}{\partial y}+\frac{T_{y}}{\rho}-\frac{c_{\mathrm{f}} \tilde{v}}{(\zeta+h)}\left(u^{2}+v^{2}\right)^{1 / 2}
\end{aligned}
$$

where $\tilde{u}=(\zeta+h) u$ and $\tilde{v}=(\zeta+h) v$ are new prognostic variables and $(\zeta+h)$ represents the total depth of the basin. 


\section{Formulation of the Model}

The model covers an analysis area spanning $\left[83.5{ }^{0} \mathrm{E}\right.$ to $\left.94.5{ }^{0} \mathrm{E}\right]$ and $\left[18{ }^{0} \mathrm{~N}\right.$ to $\left.23{ }^{0} \mathrm{~N}\right]$. It has a fixed eastern boundary at approximately $250 \mathrm{~km}$ from India's east coast, at $x=b_{2}(y)$. The treatment of coastal boundaries involves a procedure leading to a realistic curvilinear representation of both the western and eastern sides of the Bay of Bengal. The model also incorporates increased spatial resolution adjacent to the coastline. The resolution of the Head of the Bay of Bengal model is high $(\Delta x=3.7 \mathrm{~km}, \Delta \mathrm{y}=3.5$ $\mathrm{km}$, and $\Delta \mathrm{t}=60 \mathrm{sec}$ ). For the formulation of the model, a system of rectangular Cartesian coordinates $\mathrm{u}, v, w$ is used. The western coastal boundary (the east coast of India) is situated at $x=b_{1}(y)$ and the eastern coastal boundary is located at $x=b_{2}(y)$. Southern open sea and northern coastal boundaries are at $y=0$ and $L$, respectively.

After neglecting barometric forcing terms, the governing Equations (25) to (27) reduce to

$$
\begin{gathered}
\frac{\partial \zeta}{\partial t}+\frac{\partial \tilde{u}}{\partial x}+\frac{\partial \tilde{v}}{\partial y}=0 \\
\frac{\partial \widetilde{u}}{\partial t}+\frac{\partial}{\partial x}(u \tilde{v})+\frac{\partial}{\partial y}(u \tilde{v})-f \tilde{v}=-g(\zeta+h) \frac{\partial \zeta}{\partial x}+\frac{T_{x}}{\rho}-\frac{c_{\mathrm{f}} \tilde{u}}{(\zeta+h)}\left(u^{2}+v^{2}\right)^{1 / 2} \\
\frac{\partial \tilde{v}}{\partial t}+\frac{\partial}{\partial x}(u \tilde{v})+\frac{\partial}{\partial y}(u \tilde{v})+f \tilde{u}=-g(\zeta+h) \frac{\partial \zeta}{\partial y}+\frac{T_{y}}{\rho}-\frac{c_{\mathrm{f}} \tilde{v}}{(\zeta+h)}\left(u^{2}+v^{2}\right)^{1 / 2}
\end{gathered}
$$

\section{Boundary conditions of the Model}

For the Bay of Bengal, all coastal boundaries (except $y=0$ ) are coastal sidewalls. The condition of zero normal velocity at these boundaries is given by

$$
\begin{gathered}
u-v \frac{\partial b_{1}}{\partial y}=0 \text { at } x=b_{1}(y) \\
u-v \frac{\partial b_{2}}{\partial y}-\left(\frac{g}{h}\right)^{1 / 2} \zeta=0 \text { at } x=b_{2}(y)
\end{gathered}
$$

and

$$
v-\left(\frac{g}{h}\right)^{1 / 2} \zeta=0 \text { at }=L
$$

Conceptually, a radiation condition is applied at the southern open-sea boundary to yield

$$
v+\left(\frac{g}{h}\right)^{1 / 2} \zeta=0 \text { at } y=0
$$

\section{Coordinate transformation}

To simplify the numerical treatment of an irregular boundary configuration we introduce a coordinate transformation that is similar to that used by Johns et al. (1981) in [19] and which is based on a new set of independent variables $\xi, y$, and $t$, where

$$
\begin{aligned}
& \xi=\frac{x-b_{1}(y)}{b(y)} \\
& b(y)=b_{2}(y)-b_{1}(y)
\end{aligned}
$$

Thus, the western and eastern boundaries correspond to $\xi=0$ and 1 , respectively. Taking $\xi, y, t$ as our new, independent coordinates, Equations (28) to (30) may be rewritten as 


$$
\begin{gathered}
\frac{\partial}{\partial t}(b \zeta)+\frac{\partial}{\partial \xi}[b H U]+\frac{\partial}{\partial y}(b H v)=0 \\
\frac{\partial \widetilde{u}}{\partial t}+\frac{\partial}{\partial \xi}(U \tilde{u})+\frac{\partial}{\partial y}(v \tilde{u})-f \tilde{v}=-g(\zeta+h) \frac{\partial \zeta}{\partial \xi}+\frac{b T_{x}}{\rho}-\frac{c_{\mathrm{f}} \tilde{u}}{(\zeta+h)}\left(u^{2}+v^{2}\right)^{1 / 2} \\
\frac{\partial \tilde{v}}{\partial t}+\frac{\partial}{\partial \xi}(U \tilde{v})+\frac{\partial}{\partial y}(v \tilde{v})+f \tilde{u}=-g(\zeta+h)\left[b \frac{\partial \zeta}{\partial y}-\left(\frac{\partial b_{1}}{\partial y}+\xi \frac{\partial b}{\partial y}\right) \frac{\partial \zeta}{\partial \xi}\right] \\
+\frac{b T_{y}}{\rho}-\frac{c_{\mathrm{f}} \tilde{v}}{(\zeta+h)}\left(u^{2}+v^{2}\right)^{1 / 2}
\end{gathered}
$$

where

$U=\frac{1}{b(y)}\left[u-\left(\frac{\partial b_{1}}{\partial y}+\xi \frac{\partial b}{\partial y}\right) v\right]$

$\tilde{u}=H b u, \tilde{v}=H b v$, and $H=\zeta+h$

Now the boundary conditions take the form

$U=0 \quad$ at $\xi=0$

$b U-\left(\frac{g}{h}\right)^{\frac{1}{2}} \zeta=0 \quad$ at $\xi=1$

\section{Numerical procedure}

We define discrete coordinate points in the $(\xi-y)$ plane, i.e.

$$
\xi=\xi_{i}=(i-1) \Delta \xi, i=1,2,3, \ldots, m, \Delta \xi=1 /(m-1)
$$

and

$$
y=y_{i}=(j-1) \Delta y, j=1,2,3, \ldots, n, \Delta y=L /(n-1)
$$

and a sequence of time steps as

$$
t=t_{p}=p \Delta t, p=0,1,2,3, \ldots, \ldots
$$

Any variable $X$, at grid point $(i, j)$ can be represented by

$$
X\left(\xi_{i}, y_{j}, t_{p}\right)=X_{i, j}^{p}
$$

Finite-difference operators are defined as

$$
\begin{aligned}
& \frac{\partial X}{\partial t}=\delta_{t} X=\left(X_{i, j}^{p+1}-X_{i, j}^{p}\right) / \Delta t \\
& \frac{\partial X}{\partial \xi}=\delta_{\xi} X=\left(X_{i+1, j}^{p}-X_{i-1, j}^{p}\right) /(2 \Delta \xi) \\
& \frac{\partial X}{\partial y}=\delta_{y} X=\left(X_{i, j+1}^{p}-X_{i, j-1}^{p}\right) /(2 \Delta y)
\end{aligned}
$$

averaging operators as

$$
\begin{aligned}
& \bar{X}^{\xi}=\frac{1}{2}\left(X_{i+1, j}^{p}+X_{i-1, j}^{p}\right) \\
& \bar{X}^{y}=\frac{1}{2}\left(X_{i, j+1}^{p}+X_{i, j-1}^{p}\right) \\
& \bar{X}^{\xi y}=\bar{X}^{y}
\end{aligned}
$$

and a shift operator as 


$$
E_{t} X=X_{i, j}^{p+1}
$$

Equation (37), the equation of continuity, is then discretized as

$$
\Delta_{t}(b \zeta)+\delta_{\xi}\left[b\left(\bar{\zeta}^{\xi}+h\right) U\right]+\delta_{y} \tilde{v}=0
$$

This equation results in an updating procedure to compute the elevation at the interior $\zeta$ points and is consistent with mass conservation in the system. The elevations at $j=1$ and $i=2,4, \ldots, m-2$ are determined by Equation (34). In practice, this is applied at $j=2$ and replaced by

$$
\frac{1}{2}\left(\frac{g}{h_{i, 2}}\right)^{\frac{1}{2}}\left(\zeta_{i, 1}^{p+1}+\zeta_{i, 3}^{p+1}\right)+v_{i, 2}^{p}=0
$$

thus leading to an updating procedure for the elevation at the western open-sea boundary of the form

$$
\zeta_{i, 1}^{p+1}=-\zeta_{i, 3}^{p+1}-2\left(\frac{h_{i, 2}}{g}\right)^{\frac{1}{2}} v_{i, 2}^{p}
$$

Similarly, the elevations at $j=n$ and $i=2,4, \ldots, m-2$ are determined by Equation (33), which takes the form

$$
\zeta_{i, n}^{p+1}=-\zeta_{i, n-2}^{p+1}+2\left(\frac{h_{i, n-1}}{g}\right)^{\frac{1}{2}} v_{i, n-1}^{p}
$$

The elevation along the southern open-sea boundary, $\xi=1$, is determined by Equation (43), which yields

$$
\zeta_{m, j}^{p+1}=-\zeta_{m-2, j}^{p+1}+2\left(\frac{h_{m-1, j}}{g}\right)^{\frac{1}{2}} b_{j} U_{m-1, j}^{p}
$$

This equation returns updated elevations for $i=m$. Elevations along the coast at $i=1$ are not carried through in our computational scheme. These are determined by linear extrapolation from the adjacent $\zeta$ points using

$$
\zeta_{1, j}^{p+1}=\frac{1}{2}\left(3 \zeta_{2, j}^{p+1}-\zeta_{4, j}^{p+1}\right)
$$

Equation (38) is discretized as

$$
\begin{aligned}
\Delta_{t} \tilde{u}+\delta_{\xi}\left[\bar{U}^{\xi} \overline{\tilde{u}}^{\xi}\right]+\delta_{y}\left[\bar{v}^{\xi} \overline{\tilde{u}}^{y}\right] & -f \overline{\tilde{v}}^{\xi y}=-g E_{t}\left[\left(\bar{\zeta}^{\xi}+h\right) \delta_{\xi} \zeta\right]+\frac{b T_{x}}{\rho} \\
& -\frac{c_{\mathrm{f}}\left[u^{2}+(\bar{v} \xi y)^{2}\right]^{\frac{1}{2}} E_{t}(\widetilde{u})}{E_{t}\left(\bar{\zeta}^{\xi}+h\right)}
\end{aligned}
$$

It is used to update $\tilde{u}$ at the $u$ points. Updated values of $u$ may then be obtained by applying Equation (41),

$$
u_{i, j}^{p+1}=\frac{\widetilde{u}_{i, j}^{p+1}}{b_{j}\left(\zeta_{i, j}^{\overline{P+1} \xi}+h_{i, j}\right)}
$$

Equation (57) is applied for $i=3,5, \ldots, m-1$ and $j=3,5, \ldots, n-2$. With the help of Equation (42) the boundary value of $U$ at $i=1$, referenced by the averaging operator in Equation (57) is identically zero. When applied at $i=m-1$, the averaging operator in 
Equation (58) references values of $U$ outside the analysis area and, to overcome this difficulty, an appropriate one-sided extrapolation of $\delta_{\xi}$ is used. Similarly, when Equation (43) is applied at $j=3$ and $j=n-2$, the averaging operator references values of $u$ at $j=1$ and $j=n$ and, at these positions, we also use a one-sided definition of $\delta_{y}$. This way, values of $\tilde{u}$ ( or $u$ ) may be updated at all interior $u$ points.

A similar discretization scheme is applied to Equation (39), whose finite-difference form may be written as

$$
\begin{gathered}
\Delta_{t} \tilde{v}+\delta_{\xi}\left[\bar{U}^{y} \overline{\tilde{v}}^{\xi}\right]+\delta_{y}\left[\bar{v}^{y} \overline{\tilde{v}}^{y}\right]+E_{t}\left[f \overline{\tilde{u}}^{\xi y}\right] \\
=-g E_{t}\left[b\left(\bar{\zeta}^{y}+h\right) \delta_{y} \zeta-\left(\frac{\partial b_{1}}{\partial y}+\xi \frac{\partial b}{\partial y}\right)\left(\bar{\zeta}^{y}+h\right) \delta_{\xi} \bar{\zeta}^{\xi y}\right] \\
+\frac{b T_{y}}{\rho}-\frac{c_{\mathrm{f}}\left[\left(\bar{u}^{\xi y}\right)^{2}+v^{2}\right]^{\frac{1}{2}} E_{t}(\tilde{v})}{E_{t}\left(\bar{\zeta}^{y}+h\right)}
\end{gathered}
$$

This equation is used to update $\tilde{v}$ at the $v$ points. Updated values of $v$ may then be determined by applying Equation (41)

$$
v_{i, j}^{p+1}=\frac{\tilde{v}_{i, j}^{p+1}}{b_{j}\left(\zeta_{i, j}^{\bar{P}+1} y+h_{i, j}\right)}
$$

Equation (59) is applied for $i=2,4, \ldots, m-2$ and $j=2,4, \ldots, n-1$. One-sided definitions of $\delta_{y}$ are used when $j=2$ and $j=n-1$. Once $u$ and $v$ have been updated, $U$ may also be updated using Equation (40), whose discretized form may be written as

$$
U=\frac{1}{b}\left[u-\left(\frac{\partial b_{1}}{\partial y}+\xi \frac{\partial b}{\partial y}\right) \bar{v}^{\xi} y\right]
$$

\section{Results and discussion}

In this paper, we analyzed the characteristics of the severe cyclone Sidr-2007, whose track is shown in (Fig. 1). Adopting an actual duration of $18 \mathrm{hr}$, we obtained a simulated peak surge value of $6.20 \mathrm{~m}$, a radius of maximum wind $\left(R_{\max }\right)$ of $40 \mathrm{~km}$, and a pressure drop of $64 \mathrm{hPa}$ (Fig. 2). However, in the fast-moving case, the cyclone's duration was 12 $\mathrm{hr}$, while we obtained a maximum surge value of $4.28 \mathrm{~m}$ (Fig. 3). We also considered a slow-moving alternative that lasted $24 \mathrm{hr}$. The simulated peak surge for a cyclone of the same intensity as Sidr-2007 was 6.34 m (Fig. 4). Therefore, we conclude that the surge heights of severe cyclones are comparatively lower for faster-moving systems and higher for more slowly moving systems. 


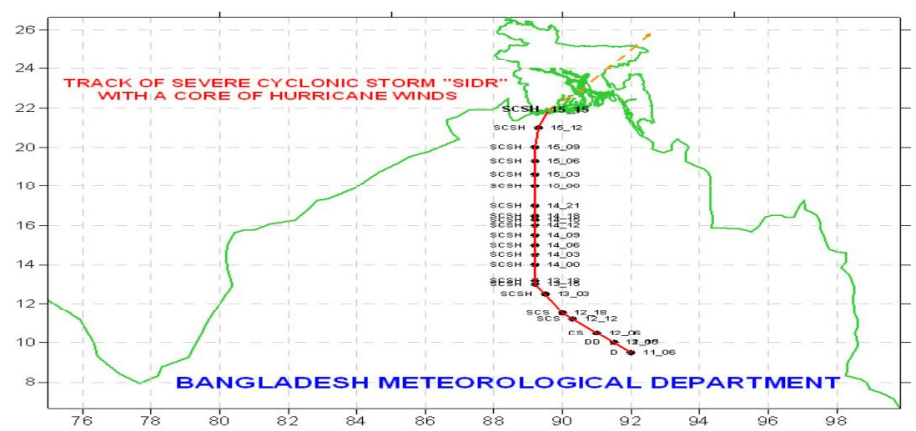

Figure 1: Track of the severe cyclone Sidr-2007. (S)CS(H): (Severe) Cyclonic Storm (Hurricane). (DD): (Deep) Depression

For cyclone land falls in different coastal zones, we obtained different results for the surge heights of severe cyclones of the same intensity as Sidr-2007. We considered three different coastal shapes, in particular convex, concave, and complex. Depending on the geometric shape, the resulting surge height varied significantly. In the eastern coastal zone, particularly at our investigations point location of $\left(22.50^{\circ} \mathrm{N}, 91.80{ }^{\circ} \mathrm{E}\right)$, the coastal shape is convex and the simulated peak-surge value was $3.84 \mathrm{~m}$ (Fig. 5). In the central coastal zone, specifically at $\left(22.20{ }^{\circ} \mathrm{N}, 90.70{ }^{\circ} \mathrm{E}\right)$, the coastal shape is concave and the simulated maximum surge value was $4.20 \mathrm{~m}$ (Fig. 6). Similarly, in the western coastal zone, at $\left(21.90^{\circ} \mathrm{N}, 89.20^{\circ} \mathrm{E}\right)$, the coastal shape is complex and our simulated maximum surge was $3.85 \mathrm{~m}$ (Fig. 7). Thus, a concave coast will experience a significantly higher storm surge than a convex coast.

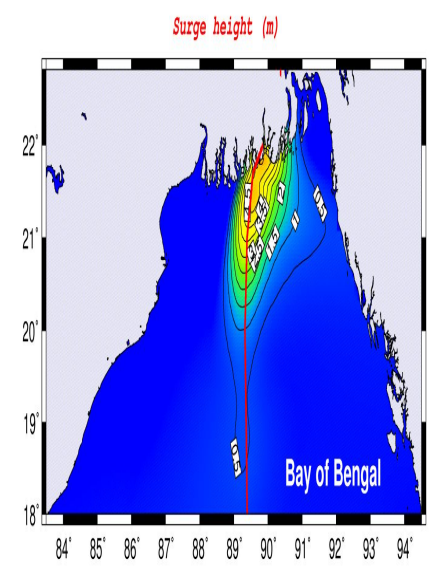

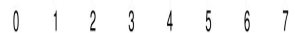

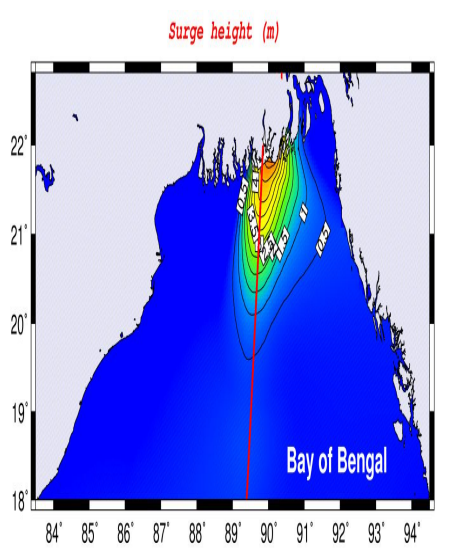

$\begin{array}{llllllll}0 & 1 & 2 & 3 & 4 & 5 & 6 & 7\end{array}$

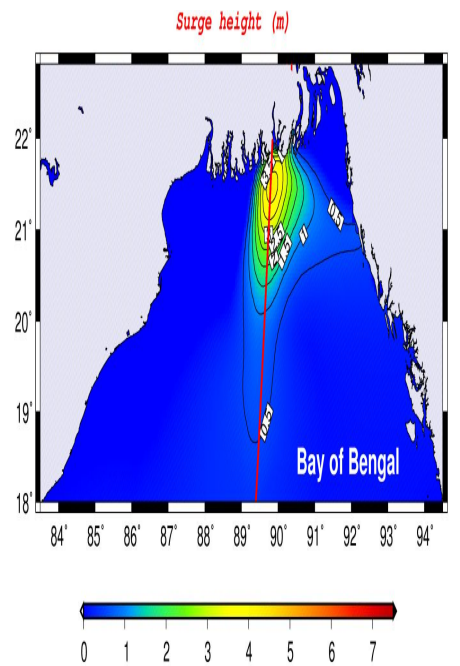

Figure 3: Storm surge for fastmoving cyclone of similar intensity as Sidr-2007.

Figure 4: Storm surge for slow- moving cyclone of similar intensity as Sidr-2007 severe cyclone Sidr-2007 


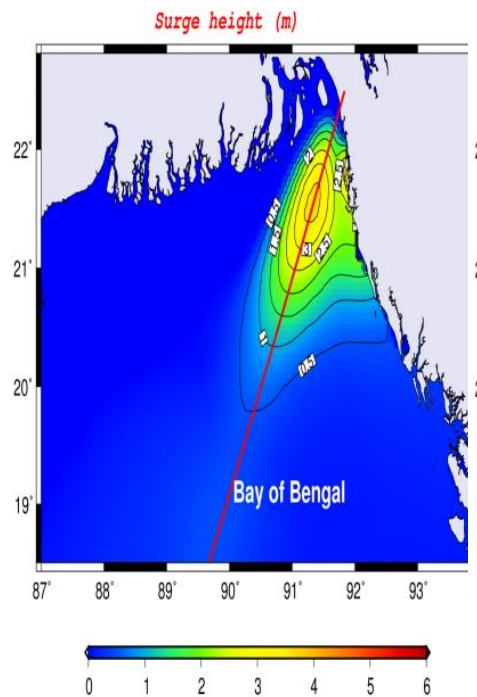

Figure 5: Storm surge for a cyclone of the same intensity as Sidr-2007, land fall in the eastern coastal zone
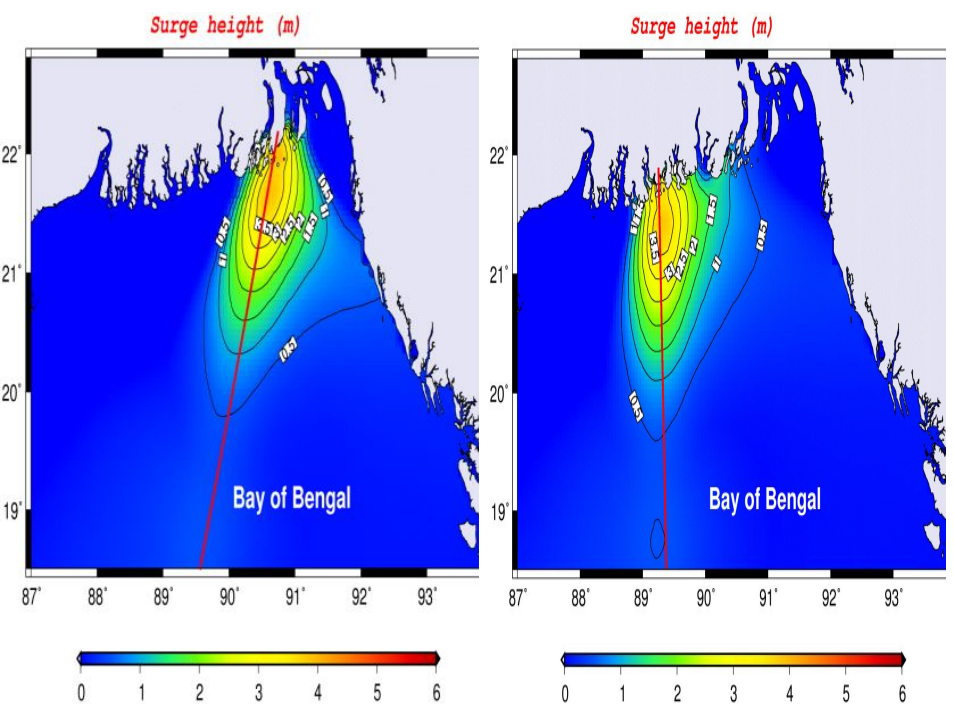

Figure 6: Storm surge due to a cyclone of the same intensity as Sidr-2007, making land fall in the western coastal zone
Figure 7: Storm surge due to a cyclone of the same intensity as Sidr-2007, making making land fall in the central zone.

\section{Conclusion}

The severe cyclone Sidr-2007, which developed in the northern part of the Bay of Bengal, was selected to simulate characteristics such as its structure, intensity, and movement. The location of the highest storm surge depends on the duration and angle of landfall, as shown in this paper. Through this simulation, it was shown that the central coastal zone is the most vulnerable region for storm surges in Bangladesh because of its physical shape. It was also shown that the resulting storm surge is higher for slowly moving storms than for the actual system, and lowers for fast-moving systems. The location of the highest surge depends predominantly on the angle of landfall. Cyclones of similar intensity generate surges of different amplitudes, depending upon the angle of landfall at a particular location. A concave coast will experience a higher surge than a convex coastal zone.

\section{REFERENCES}

[1] Ali, A., 1996: Vulnerability of Bangladesh to Climate Change and Sea Level Rise through tropical cyclones and storm surges. Water, Air and Soil Pollution, 92, 171-179.

[2] Ali, A., Rahman, H., Sazzard, S., and Choudhary, H., 1997: River discharge, storm surges and tidal interaction in the Meghna river mouth in Bangladesh. Mausam. 48, 531-540.

[3] Chittibabu, P., 1999: Development of storm surge prediction models for the Bay of Bengal and the Arabian Sea. Ph.D. Thesis, IIT Delhi, India, 200pp.

[4] Das, P. K., 1980: Storm surges - A review of current problems. WMO workshop on storm surges, 10-15 Nov. 1980, Rangoon, Burma. 
[5] Das, P. K., 1981: Storm surges in the Bay of Bengal. Proc. Ind. Acad. Sci. (Eng. Sci.), 4, 269-276.

[6] Das, P. K., Dube, S. K., Mohanty, U. C., Sinha, P. C., and Rao, A. D., 1983. Numerical simulation of the surge generated by the June 1982 Orissa cyclone. Mausam, 34, 359-366.

[7] Debsarma, S. K., 1997: Visualization of May 1997 storm surge by using IIT model, Proc. SAARC seminar on Tropical Cyclones and Storm Surges in the South Asian Region, 20-22 Dec. 2003 (Dhaka, Bangladesh), p. 23-48.

[8] Debsarma, S. K., 2009: Simulations of storm surges in the Bay of Bengal, Marine Geodesy. 32, 178-198.

[9] Dube, S. K., and Singh, M. P., 1982: Storm surges in India and neighborhood - The problems and numerical simulation. WMO Workshop on Monsoon Dynamics, Dhaka, 11 Jan.-15 Feb. 1982.

[10] Dube, S. K., Sinha, P. C. and Roy, G. D., 1984: Storm surges in Bangladesh - The problem and numerical simulation, Vayu Mandal, in press.

[11] Flather, R. A., and Davies, A. M., 1976: Note on a preliminary scheme for storm surge prediction using numerical models. Quart. J. R. Meteor. Soc., 102, 123-132.

[12] Ghosh, S. K., 1983: Probable maximum storm surge on the coast of India and Bangladesh. Invited talk at $27^{\text {th }}$ ISTAM Congress, Dec. 1983, Jadavpur Univ., Calcutta, India.

[13] Ghosh, S. K., and Chanda, B. N., 1983: A scheme for objective prediction of storm surges on the Saurashtra coast. Communicated to $2^{\text {nd }}$ Asian Congress of Fluid Mechanics, 25-29 Oct. 1983, Beijing, China.

[14] Heaps, N. S., and Jones, J. E., 1979: Recent storm surges in the Irish Sea. Marine Forecasting (J. C. Nihoul, Ed.), Elsevier Ocean. Ser., 285-319.

[15] Hoque, A., 2008: Studies of water level rise by entrained air in the surf zone, Exp. Therm. Fluid Sci. 32, 973-979.

[16] Roy, G. D., 1999: Inclusion of off-shore islands in a transformed coordinate shallow water model along the coast of Bangladesh. Environ. Int., 25, 67-74.

[17] Sarkar, C. S., Islam, A., and Debsarma, S. K., 2011: Coastal geometry and storm surge model for the Bay of Bengal. Proc. Int'l Conf. on Environmental Technology and Construction Engineering for Sustainable Development, pp. 74-75.

[18] Sinha, P. C., Dube, S. K., Rao, A. D., and Rao, G. S., 1984: Numerical simulation of the surge generated by the November 1982 Gujrat cyclone. Vyau Mandal, in press.

[19] Johns, B., 1981: Numerical simulation of storm surges in the Bay of Bengal. In: Monsoon Dynamics, ed. J. Lighthill and R. P. Pearce, pp. 690-705. New York: Cambridge Univ. Press.

[20] Johns, B., Dube, S. K., Sinha, P. C., Mohanty, U. C., and Rao, A. D., 1983: Simulation of storm surges using a three-dimensional numerical model: An application to the 1977 Andhra Cyclone. Quart. J. R. Meteor. Soc., 109, 211-224. 